\title{
DISFUNÇÕES TEMPOROMANDIBULARES: SINAIS, SINTOMAS E ABORDAGEM MULTIDISCIPLINAR
}

\author{
Temporomandibular Disorders: signs, symptoms \\ and multidisciplinary approach
}

Mariana Del Cistia Donnarumma ${ }^{(1)}$, Carlos Alberto Muzilli ${ }^{(2)}$, Cristiane Ferreira ${ }^{(3)}$, Kátia Nemr ${ }^{(4)}$

\section{RESUMO}

Objetivo: verificar perfil, queixa e principais sinais e sintomas de uma amostra de pacientes com disfunção temporomandibular que estiveram ou estão em tratamento ortodôntico e observar a ocorrência de atendimentos multidisciplinares. Métodos: foram coletados dados de 125 prontuários em uma clínica odontológica da cidade de Sorocaba e os itens analisados nos prontuários foram: sexo, idade, profissão, queixa, três principais sinais e sintomas dos pacientes e se houve encaminhamento para avaliação fonoaudiológica, fisioterápica e psicológica. Resultados: predominância feminina, sendo 107 mulheres (85,6\%) e 18 homens (14,4\%). Média de idade de 35 anos, sendo a menor idade 14 anos e a maior 74 anos. Relação da disfunção temporomandibular com as profissões: 43 (34,4\%) eram profissionais com vínculo empregatício. Queixa trazida pelo paciente: dor na região da articulação temporomandibular e masseter: 86 - (68,8\%). Três principais sinais e sintomas observados na avaliação ortodôntica: dor na região da articulação temporomandibular e masseter: 98 - 78,4\%; estalos unilaterais: $55-44 \%$ e travamento: $23-18,4 \%$. Conduta de encaminhamentos: fonoaudiologia 59 (47,2\%); fisioterapia 40 (32\%) e psicologia 53 (42,4\%). Conclusão: na amostra pesquisada, a prevalência de casos de disfunção temporomandibular foi maior no sexo feminino, com queixa de dor. Os principais sinais e sintomas foram: dor, estalo unilateral e travamento e houve encaminhamento para atendimentos multidisciplinares nas áreas de Fonoaudiologia, Fisioterapia e Psicologia.

DESCRITORES: Síndrome da Disfunção da Articulação Temporomandibular; Dor Facial

\section{INTRODUÇÃO}

A articulação temporomandibular (ATM) é a única articulação móvel do crânio. É considerada a mais complexa do corpo humano, por duas razões: é a única que permite movimentos rotacionais e translacionais, devido à articulação dupla do côndilo.

(1) Fonoaudióloga em Clínica Especializada em Audiometria e Terapias; Especializanda em Motricidade Orofacial pelo CEFAC - Saúde e Educação.

(2) Dentista; Professor da Universidade Paulista - Sorocaba e São Leopoldo Mandique - Campinas; Doutor em Próteses Dentárias pela Universidade de São Paulo.

(3) Fonoaudióloga da Prefeitura de Mauá-SP; Especialista em Linguagem, Especializanda em Motricidade Orofacial pelo CEFAC - Saúde e Educação.

(4) Fonoaudióloga; Docente da Universidade de São Paulo; Doutora em Psicologia Social pela Universidade de São Paulo.

Conflito de interesses: inexistente
Além disto, existem duas articulações conectadas a um único osso, a mandíbula, as quais funcionam simultaneamente ${ }^{1}$. Para que a articulação temporomandibular funcione de forma adequada, a própria articulação temporomandibular, a oclusão dental e o equilíbrio neuromuscular devem relacionar-se harmonicamente ${ }^{2}$.

O termo disfunção temporomandibular (DTM) é utilizado para reunir um grupo de doenças que acometem os músculos mastigatórios, ATM e estruturas adjacentes ${ }^{3}$. As DTMs podem ser classificadas em dois grandes subgrupos: as de origem articular, ou seja, aquelas em que os sinais e sintomas estão relacionados à ATM; e as de origem muscular nas quais os sinais e sintomas relacionamse com a musculatura estomatognática ${ }^{4}$. A DTM tem etiologia multifatorial ${ }^{2,5-10}$ e está relacionada com fatores estruturais, neuromusculares, oclusais ${ }^{11}$ (perdas dentárias, desgaste dental, próteses mal adaptadas, cáries, restaurações inadequadas 
entre outras), psicológicos (devido a tensão há um aumento da atividade muscular que gera espasmo e fadiga), hábitos parafuncionais (bruxismo, onicofagia, apoio de mão na mandíbula, sucção digital ou de chupeta) ${ }^{2}$ e lesões traumáticas ou degenerativas da ATM ${ }^{12}$.

As DTM têm interpretação muito ampla e descrevem uma população geral de pacientes sofrendo de disfunção dos músculos e articulações da mandíbula, usualmente dolorosa ${ }^{13}$. Quando presente, a DTM caracteriza-se por dores nas articulações temporomandibulares e nos músculos mastigatórios, sendo a dor o sintoma mais comum e as mulheres são mais afetadas que os homens numa proporção de 4:1 7 .

Normalmente essa disfunção afeta tão enfaticamente a população que num estudo recente, os autores concluíram que a dor da DTM tem um impacto negativo na qualidade de vida do paciente, prejudicando as atividades do trabalho $(59,09 \%)$, da escola $(59,09 \%)$, o sono $(68,18 \%)$ e o apetite/ alimentação $(63,64 \%)$ nos sujeitos pesquisados ${ }^{14}$. Os sintomas auditivos referidos por pacientes com DTM são: dores de ouvido (otalgia), sensação de plenitude auricular, sensação de diminuição de acuidade auditiva, zumbidos, tonturas e vertigens ${ }^{11}$. Outros sintomas são: limitação dos movimentos mandibulares, oclusão estática e dinâmicas anormais ${ }^{11}$ e também pode haver a presença de ruídos articulares (como estalido e/ou crepitação). O estalo pode ou não ser acompanhado de dor ${ }^{12}$ e o estalido (clicking) duplo, na abertura e fechamento mandibular, caracteriza-se por deslocamento do disco articular com redução, e a articulação silenciosa assintomática e limitação na abertura indicam deslocamento do disco articular sem redução. A crepitação freqüentemente indica uma artrose ${ }^{10}$.

Para uma correta indicação terapêutica, a avaliação de todos os possíveis sintomas juntamente com o trabalho em equipe é fundamental. Cirurgiões-dentistas, fisioterapeutas, fonoaudiólogos, além de psicólogos, otorrinolaringologistas, neurologistas e clínicos da dor devem conjuntamente avaliar os possíveis fatores causais e, cada qual em sua área de atuação, intervir ${ }^{6}$.

Sabendo que a DTM está inserida no campo de atuação fonoaudiológica, os objetivos da pesquisa foram: verificar perfil, queixa e principais sinais e sintomas de uma amostra de pacientes com disfunção temporomandibular que estiveram ou estão em tratamento ortodôntico e verificar a ocorrência de atendimentos multidisciplinares.

\section{MÉTODOS}

Foi realizada uma pesquisa retrospectiva, por resgate de prontuários de indivíduos com alteração temporomandibular, que passaram ou ainda estavam em tratamento, em uma clínica odontológica da cidade de Sorocaba. A amostra foi composta de 125 prontuários.

As variáveis analisadas foram: sexo, idade, profissão, queixa, três principais sinais e sintomas dos pacientes e a presença de encaminhamento para avaliação fonoaudiológica, fisioterápica e psicológica.

Para classificação das profissões e a divisão das classes profissionais utilizou-se as categorias do Ministério do Trabalho e Emprego, na categoria CBO de 2002 (Classificação Brasileira de Ocupações), utilizando a categoria dos grandes grupos de profissões regulamentadas (GG). Para completar a classificação foi utilizado juntamente a CIUO (Classificação Internacional Uniforme de Ocupações), no qual todas as profissões nacionais estão representadas pelas sigla CIUO - 88 (1).

Os critérios de exclusão foram: crianças menores de 12 anos de idade, com DTM; traumas craniofaciais e uso de prótese total. Já os critérios de inclusão utilizados foram: estar em acompanhamento ou ter sido atendido por um dos ortodontistas, independentemente do tratamento, incluindo na amostra as altas e desistências.

O Comitê de Ética em Pesquisa do Centro de Especialização em Fonoaudiologia Clínica aprovou o projeto desta pesquisa sob número 137/07.

Os achados foram apresentados em frequências absolutas e relativas. Para a descrição das variáveis foram utilizadas: variáveis paramétricas (média, desvio-padrão, mínimo e máximo) e variáveis não-paramétricas (frequência e respectivo percentual). O programa utilizado foi SPSS (Statistical Package for Social Sciences), em sua versão 13.0.

\section{RESULTADOS}

Após a análise dos 125 prontuários pode-se constatar que houve predominância feminina, sendo 107 mulheres $(85,6 \%)$ e 18 homens (14,4\%) (Figura 1). Quanto à idade obteve-se uma média de 35 anos, sendo a menor idade 14 anos e a maior 74 anos (Tabela 1).

No que se refere à relação profissões e DTM observou-se a seguinte incidência: 43 (34,4\%) profissionais com vínculo empregatício; 24 pessoas $(19,2 \%)$ profissionais do lar; $21(16,8 \%)$ autônomos (de variadas áreas); 12 (9,6\%) estudantes (de diferentes áreas); 8 (6,4\%) profissionais da área 


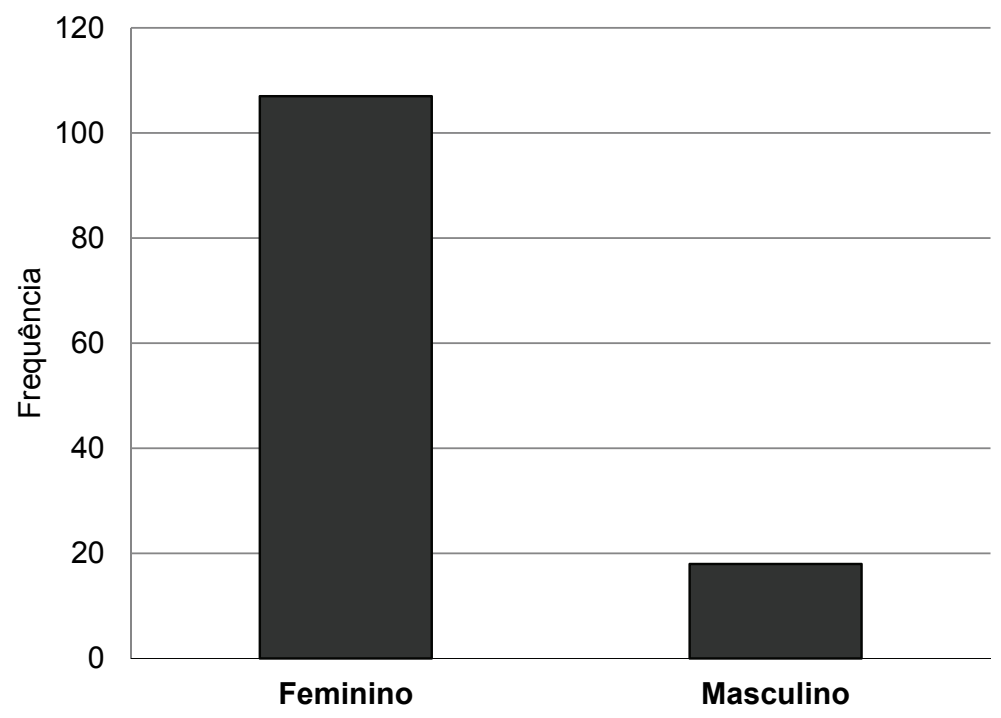

Figura 1 - Distribuição em freqüências absolutas da amostra em relação ao sexo

Tabela 1 - Faixa etária da amostra com DTM, com idade mínima, máxima, média e desvio padrão

\begin{tabular}{cccccc}
\hline Variável & $\mathbf{n}$ & Mínimo & Máximo & Média & Desvio-padrão \\
\hline Idade & 125 & 14,00 & 74,00 & 35,62 & 12,25 \\
\hline
\end{tabular}

n- número absoluto

da saúde; $10(8 \%)$ professores de variadas matérias, do ensino infantil à graduação; cinco (4,0\%) aposentados; e duas $(1,6 \%)$ outras profissões, que e que somadas não tiveram número expressivo (Tabela 2).

Outra variável analisada foi quanto às queixas trazidas pelos pacientes que representaram: dor por $86(68,8 \%)$, estalo por $43(34,4 \%)$, travamento por $13(10,4 \%)$, diminuição de abertura de boca por $10(8 \%)$, bruxismo por cinco (4\%), fadiga muscular por quatro $(3,2 \%)$, dificuldade de mastigação por quatro $(3,2 \%)$, morder lábios e bochechas por dois $(1,6 \%)$ e apertamento por dois $(1,6 \%)$ (Tabela 3$)$.

Foram averiguados os principais sinais e sintomas observados na avaliação ortodôntica, resultando: dor na região da ATM e masseter por 98 $(78,4 \%)$, estalo unilateral por $55(44 \%)$, travamento por $23(18,4 \%)$, diminuição de abertura de boca por 15 (12\%), estalo bilateral por $15(12 \%)$, dor de cabeça por $15(12 \%)$, dor durante a mastigação por $13(10,4 \%)$, dificuldade de mastigação por $10(8 \%)$, bruxismo por nove $(7,2 \%)$, fadiga muscular por seis $(4,8 \%)$, apertamento por quatro $(3,2 \%)$, morder lábios e bochechas por três $(2,4 \%)$, onicofagia por três $(2,4 \%)$ e zumbido por dois (1,6\%) (Figura 2$)$.

Outra variável analisada foi a conduta de encaminhamentos realizados para avaliação da
Fonoaudiologia, Fisioterapia e Psicologia, e os resultados obtidos foram: $47,2 \%$ de encaminhamentos para avaliação fonoaudiológica, $32 \%$ para avaliação fisioterápica e 42,4\% para avaliação psicológica, porém esses resultados devem ser considerados de forma especial, já que o mesmo paciente pode ter sido encaminhado para mais de uma especialidade durante o tratamento ortodôntico.

\section{DISCUSSÃO}

$\mathrm{Na}$ amostra estudada, em acordo com os achados da literatura, a freqüência de casos de DTM foi maior no sexo feminino em comparação ao sexo masculino ${ }^{5,7,12,15-21}$. A faixa etária da amostra estudada é semelhante à encontrada na literatura que mostra maior prevalência de DTM entre as idades de 20 e 40 anos 1,7,8,12,15, 16,20.

Um estudo ${ }^{15}$ descreveu as características trabalhistas de 150 pacientes de um serviço especializado em DTM e dor orofacial e os autores observaram que houve diversidade de situação profissional e verificou-se maior número de donasde-casa, estudantes e desempregados, num total de $64 \%$ da amostra, e atribuem essa prevalência ao horário de funcionamento do serviço, 
Tabela 2 - Distribuição em frequências absolutas e percentuais das profissões dos sujeitos pesquisados

\begin{tabular}{lcc}
\hline Profissão & Freqüência & Percentual \\
\hline Aposentado & 5 & $4 \%$ \\
Do lar & 24 & $19,2 \%$ \\
Estudante & 12 & $9,6 \%$ \\
Funcionário com vínculo empregatício & 43 & $34,4 \%$ \\
Professor & 10 & $8 \%$ \\
Profissional da saúde & 8 & $6,4 \%$ \\
Autônomo & 21 & $16,8 \%$ \\
Outro & 2 & $1,6 \%$ \\
\hline Total & 125 & $100 \%$ \\
\hline
\end{tabular}

Tabela 3 - Distribuição em frequências absolutas e percentuais das queixas presentes e ausentes na amostra

\begin{tabular}{|c|c|c|c|}
\hline Variável & Categoria & Frequência & Percentual \\
\hline \multirow[t]{2}{*}{ dor } & Presente & 86 & 68,8 \\
\hline & Ausente & 39 & 31,2 \\
\hline \multirow[t]{2}{*}{ estalo } & Presente & 43 & 34,4 \\
\hline & Ausente & 82 & 65,6 \\
\hline \multirow[t]{2}{*}{ travamento } & Presente & 13 & 10,4 \\
\hline & Ausente & 112 & 89,6 \\
\hline \multirow[t]{2}{*}{ diminuição da abertura de boca } & Presente & 10 & 8 \\
\hline & Ausente & 115 & 92 \\
\hline \multirow[t]{2}{*}{ hábito Deletério Bruxismo } & Presente & 5 & 4 \\
\hline & Ausente & 120 & 96 \\
\hline \multirow[t]{2}{*}{ fadiga na Muscular } & Presente & 4 & 3,2 \\
\hline & Ausente & 121 & 96,8 \\
\hline \multirow[t]{2}{*}{ dificuldade de mastigação } & Presente & 4 & 3,2 \\
\hline & Ausente & 121 & 96,8 \\
\hline \multirow[t]{2}{*}{ hábito Deletério morde lábios e bochechas } & Presente & 2 & 1,6 \\
\hline & Ausente & 123 & 98,4 \\
\hline \multirow[t]{3}{*}{ hábito Deletério apertamento } & Presente & 2 & 1,6 \\
\hline & Ausente & 123 & 98,4 \\
\hline & Total & 125 & 100,0 \\
\hline
\end{tabular}


por apresentarem maior disponibilidade para comparecer ao ambulatório.

Em estudo ${ }^{16}$ no qual avaliou-se a incidência de hábitos deletérios e posturais, por meio de fichas clínicas e avaliação postural, em 191 pacientes portadores de DTM, pode-se observar que houve grande freqüência de hábitos como colocar a mão no queixo $(73,5 \%)$, apertar dentes $(59,7 \%)$ e morder objetos $(43,5 \%)$ e também $46 \%$ dos pacientes apresentavam flexão de cabeça e $70,7 \%$ postura atípica dos ombros; houve também associação entre DTM e o exercício de profissões que exigem um maior esforço muscular em $55,5 \%$ da amostra estudada, observando-se que há interação entre hábitos deletérios, DTM e profissões que exigem esforço muscular. Outros autores ${ }^{22}$ verificaram a associação da classe econômica e do estresse com a ocorrência da DTM e não foi observada associação significante entre classe econômica e DTM, mas há associação direta com o estresse. Porém, não houve dentre a literatura pesquisada, estudos que relacionassem as profissões, o estresse e as DTMs.

No que se refere à queixa trazida pelos pacientes, este dado coincidem com os da literatura. Em trabalho realizado por outros pesquisadores, os motivos pelos quais os pacientes procuraram atendimento foram: dor de cabeça $(79 \%)$, seguidos por ruídos articulares $(44 \%)^{7}$ e dor na ATM $(41 \%)^{7,12,15,23 .}$.

Em uma pesquisa ${ }^{18}$ verificou-se a ocorrência de pacientes portadores de DTM em um serviço de Otorrinolaringologia, por meio da análise e da avaliação de um questionário dado a 221 pacientes, $21,72 \%$ dessa amostra foram considerados necessitando de tratamento para DTM. Os principais sinais e sintomas foram: dor de cabeça $(33,5 \%)$, dor no pescoço e ombro $(28,5 \%)$, dor na região do ouvido $(29 \%)$ e ruídos articulares $(25 \%)$, sintomas coincidentes aos encontrados em nosso estudo.

Outros autores 17 investigaram a associação de sintomas otológicos (otalgia, zumbido e plenitude auricular) com os achados audiológicos e os outros sinais e sintomas relacionados à desordem temporomandibular em 27 pacientes que responderam um questionário e passaram por avaliação otorrinolaringológica e audiológica. Os sinais e sintomas predominantes foram: o ruído articular, a dor muscular e a dor na região das ATMs, dado este coincidente com o de nossa amostra. Porém os sintomas otológicos foram presentes em $88,88 \%$ (59,26\% apresentavam otalgia, $74,07 \%$ zumbido e $74,07 \%$ plenitude auricular) e a conclusão que o estudo fornece é de que há relação entre desordem temporomandibular e sintomas otológicos. Isto difere dos dados encontrados em nossa amostra em que apenas $1,6 \%$ dos pacientes apresentaram sintomas otológicos, fato que pode ter ocorrido por se tratar de uma clínica odontológica e os pacientes possivelmente não relacionavam sintomas otológicos a queixa.

No que se refere às condutas de encaminhamentos para a avaliação de áreas correlacionadas a DTM, os dados obtidos apontaram uma boa demanda de encaminhamentos para avaliação fonoaudiológica $(47,2 \%)$, para avaliação fisioterápica (32\%) e avaliação psicológica (42,4\%), evidenciando a importância de um trabalho integrado entre as áreas. Essa conduta mostra que um trabalho multidisciplinar 2,6,7,11,12,15,24,25, desempenha um papel fundamental no diagnóstico e tratamento das DTMs.

\section{CONCLUSÃO}

De acordo com os resultados obtidos no presente estudo pode-se verificar: um perfil predominantemente de mulheres, com média de 35 anos de idade, com vínculo empregatício e com queixa de dor na consulta; os principais sinais e sintomas de DTM na amostra estudada foram: dor $(78 \%)$, estalo unilateral $(44 \%)$ e travamento $(18,4 \%)$ e houve encaminhamento para avaliação fonoaudiológica em $47,2 \%$ dos casos, para avaliação fisioterápica em $32 \%$ e avaliação psicológica $42,4 \%$, sendo que o mesmo paciente pode ter sido encaminhado para mais de uma especialidade. 


\section{ABSTRACT}

Purpose: to check the main signs and symptoms of a sample of patients with temporomandibular dysfunction that were or are under orthodontic treatment and observe if there was a possible multidisciplinary treatment. Methods: data from 125 medical records collected in a orthodontic clinic located in Sorocaba and the analyzed items were: gender, age, profession, complains, three main signs and symptoms of the patients and if they had been submitted to evaluation with a speech therapist, physiotherapist and psychological. Results: feminine predominance, being 107 women $(85.6 \%)$ and 18 men (14.4\%). Average of age: 35 year-old, being the smallest age 14 years and the largest 74 years. Relationship of temporomandibular dysfunction with the professions: $43-(34.4 \%)$ were professional with contract of employment. Complaint brought by the patient: pain in the area of temporomandibular articulation and masseter: 86 - (68.8\%). Three main signs and symptoms observed in the orthodontic evaluation: pain in the temporomandibular articulation and masseter area: $98-78.4 \%$; unilateral cracks: $55-44 \%$ and locking: $23-18.4 \%$. Conduct of referrals: speech therapy 59 - (47, 2\%); physiotherapy 40 (32\%) and psychology 53 (42.4\%). Conclusion: in the researched sample, the prevalence of cases related to temporomandibular dysfunction was higher in the feminine gender, with pain complaint. The main signs and symptoms were: pain, unilateral crack and locking and there was a referral for multidisciplinary cares for speech therapy, physiotherapy and psychology areas.

KEYWORDS: Temporomandibular Articulation Dysfunction Syndrome; ; Sign; Symptoms; Facial Pain

\section{REFERÊNCIAS}

1. Maydana AV. Critérios diagnósticos de pesquisa para as desordens temporomandibulares em uma população de pacientes brasileiros. [dissertação]. São Paulo: Universidade de São Paulo; 2007.

2. Quinto CA. Classificação e tratamento das disfunções temporomandibulares: qual o papel do fonoaudiólogo no tratamento dessas disfunções? Rev CEFAC. 2000; 2(2):15-22.

3. Amantea DV, Novaes AP, Campolongo GS, Barros TP. A importância da avaliação postural no paciente com disfunção temporomandibular. Acta Ortop. Bras. 2004; 12(3):155-9.

4. Farilla EE. Freqüência das parafunções orais nos diferentes subgrupos de diagnósticos de Desordens Temporomandibulares de acordo com Critérios Diagnósticos de Pesquisa em Desordens temporomandibulares (RCD/TMD). [dissertação]. São Paulo: Universidade de São Paulo; 2007.

5. Barbosa CMR, Queluz DP, Barbosa JRA, Di Hipólito Jr O. Correlação entre aparelho ortodôntico, sexo e presença de disfunções temporomandibulares. J Bras Ortop Facial. 2002; 7(39):185-92.

6. Costa LFM, Guimarães JP. Disfunções temporomandibulares: qual o papel atual do cirurgião-dentista? Rev Bras Odontol. 2002; 59(5):351-4.
7. Zanettini I, Zanettini UM. Disfunções temporomandibulares: estudo retrospectivo de 150 pacientes. Rev Cient AMECS. 1999; 8(1):9-15.

8. Pereira Jr FJ, Vieira AR, Prado R, Miasato JM. Visão geral das disfunções temporomandibulares. RGO. 2004; 52(2):117-21.

9. Coutinho MEP, Wassal T. Os efeitos do tratamento ortodôntico sobre a articulação têmporomandibular. RGO. 2003; 51(4):335-42.

10. Delboni MEG, Abrão J. Estudo dos sinais de DTM em pacientes ortodônticos assintomáticos. Rev Dental Press Ortod Ortop Facial. 2005; 10(4):88-96.

11. Felício CM. Fonoaudiologia aplicada a casos odontológicos: motricidade oral e audiologia. São Paulo: Pancast; 1999. 243 p.

12. Pereira KNF, Andrade LLS, Portal TF. Sinais e sintomas de pacientes com disfunção temporomandibular. Rev CEFAC. 2005; 7(2);221-8. 13. Manfredi APS, Silva AA, Vendite LL. Avaliação da sensibilidade do questionário de triagem para dor orofacial e desordens temporomandibulares recomendado pela Academia Americana de Dor Orofacial. Rev Bras Otorrinolaringol. 2001; 67(6):763-8.

14. Oliveira AS, Bermudez CC, Souza RA, Souza CMF, Dias EM, Castro CES, Berzin F. Impacto da dor na vida de portadores de disfunção temporomandibular. J Appl Oral Sci. 2003; 11(2):138-43. 
15. Bove SRK, Guimarães AS, Smith RL. Caracterização dos pacientes de um ambulatório de disfunção temporomandibular e dor orofacial. Rev Latino-am Enf. 2005; 13(5):686-91.

16. Cauás M, Alves IF, Tenório K, HC Filho JB, Guerra CMF. Incidência de hábitos parafuncionais e posturais em portadores de disfunção da articulação craniomandibular. Rev Cirur Traumat Buco-MaxiloFacial. 2004; 4(2):121-9.

17. Felício CM, Faria TG, SilvaMAMR, Aquino AMCM, Junqueira CA. Desordens temporomandibulares: relações entre sintomas otológicos e orofaciais. Rev Bras Otorrinolaringol. 2004; 70(6):786-93.

18. Silveira AM, Feltrin PP, Zanetti RV, Mautoni MC. Prevalência de portadores de DTM em pacientes avaliados no setor de otorrinolaringologia. Rev Bras Otorrinolaringol. 2007; 73(4):528-32.

19. Ozan F, Polat S, Kara I, Küçük D, Polat HB. Prevalence study of signs and symptoms of temporomandibular disorders in a Turkish population. J Contemp Dent Pract. 2007; 8(4):35-42. 20. Nomura K, Vitti M, Oliveira AS, Chaves TC, Semprini M, Siéssere S, Hallak C, Regalo $\mathrm{SCH}$. Use of the Fonseca's questionnare to asses the prevalence and severity of temporomandibular disorders in brazilian dental undergraduates. Braz Dent J. 2007; 18(2):163-7.

21. Bermejo-Fenoll A, Sáez-Yuguero R. Diagnóstico diferencial de los desórdenes temporomandibulares (DTM). Med Oral Patol Oral Cirur Bucal. 2005;10(5):468-9.

22. Martins RJ, Garcia AR, Garbin CAS, Sundefeld MLMM. Associação entre classe econômica e estresse na ocorrência da disfunção temporomandibular. Rev Bras Epidemiol. 2007; 10(2):215-22.

23. Cooper BC, Kleinberg I. Examination of a large patient population for the presence of symptoms and signs of temporomandibular disorders. Crânio. 2007; 25(2):114-26.

24. Poli MS, Morosini MRM, Martinelli RCPM. Abordagem interdisciplinar na disfunção temporomandibular: relato de caso. Arq Ciênc Saúde UNIPAR. 2003; 7(2):171-7.

25. Nóbrega JCM, Siqueira SRDT, Siqueira JTT, Teixeira MJ. Diferential diagnosis in atypical facial pain: a clinical study. Arq Neuro- Psiquiatr. 2007; 6(2):256-61.

DOI: 10.1590/S1516-18462010005000085

RECEBIDO EM: 31/03/2008

ACEITO EM: 18/12/09

Endereço para correspondência:

Mariana Del Cistia Donnarumma

Rua Emygdia Campolim, 120

Cidade: Sorocaba - Estado: São Paulo

CEP: 18047-626

E-mail: ma.fono@bol.com.br

ma_dcd@yahoo.com.br 